\title{
Stressing the heart of the matter: re-thinking the mechanisms underlying therapeutic effects of $n-3$ polyunsaturated fatty acids
} Ethan J. Anderson ${ }^{1,2,3 *}$ and David A. Taylor ${ }^{1}$

\author{
Addresses: ${ }^{1}$ Department of Pharmacology \& Toxicology, ${ }^{2}$ Cardiovascular Sciences, and ${ }^{3}$ East Carolina Diabetes and Obesity Institute, East Carolina \\ University, 600 Moye Blvd. Greenville, NC 27834 \\ * Corresponding author: Ethan J. Anderson (andersonet@ecu.edu) \\ FI000 Medicine Reports 2012, 4:13 (doi:10.3410/M4-13) \\ This is an open-access article distributed under the terms of the Creative Commons Attribution-Non Commercial License \\ (http://creativecommons.org/licenses/by-nc/3.0/legalcode), which permits unrestricted use, distribution, and reproduction in any medium, \\ provided the original work is properly cited. You may not use this work for commercial purposes. \\ The electronic version of this article is the complete one and can be found at: http://fl000.com/reports/m/4/I3
}

\begin{abstract}
Despite their clear therapeutic effects in coronary heart disease, use of n-3 polyunsaturated fatty acids (PUFAs) to treat other types of cardiovascular disease remains controversial, and serious obstacles exist in implementing them as a reliable and consistent drug therapy. The foremost of these is that a molecular mechanism and relevant dosages have not been firmly established in other forms of cardiovascular disease. In this brief review, we highlight the current state of knowledge regarding the mechanisms behind n-3 PUFA action in the cardiovascular system. We also propose the novel hypothesis that lipid peroxidation products derived from n-3 PUFAs may be driving much of their beneficial cardiovascular effects, particularly in the myocardium. We conclude by discussing evidence to support this hypothesis, and its possible clinical ramifications.
\end{abstract}

\section{Introduction and context}

The enormous impact of cardiovascular disease on global health and economy demands that low-cost interventions, such as altered lifestyle (e.g. diet and physical activity), be rigorously implemented for prevention and treatment. Dietary intake of n-3 PUFAs, particularly fish oil, is a propitious and therapeutically achievable intervention that has been clearly shown to have a beneficial effect on the cardiovascular system. However, it is disappointing that despite significant clinical and experimental research on n-3 PUFAs and cardiovascular disease over the past 25 years, there is still no clear molecular mechanism or appropriate dosing strategy in place, making reliable and consistent therapeutic use of n-3 PUFAs extremely difficult. Thus, a coordinated effort is needed to establish a mechanism and develop proper therapeutic paradigms to make n-3 PUFAs more amenable for use in prevention and treatment of cardiovascular disease. Here, we provide a brief overview of the debate regarding the mechanism of n-3 PUFA therapy among biomedical researchers, and present a novel hypothesis that may help reconcile this controversy and unite existing, well-characterized n-3 PUFA effects with as-yet unresolved questions.

n-3 PUFA treatment for cardiovascular disease Existing paradigms and controversy regarding mechanism Compounds that exert vasodilating, anti-inflammatory, anti-thrombotic, anti-arrhythmic and heart rate-lowering effects are all effective therapies for cardiovascular disease. While n-3 PUFAs have shown promise in virtually all of these areas, results from clinical trials have been mixed, with some showing clear benefits and others showing no change compared with placebo. Excellent comprehensive reviews of these topics are found in the recent literature $[1,2]$. To date, the mechanisms proposed to explain these beneficial cardiovascular effects have largely focused on the ability of the two predominant n-3 PUFAs in fish oil, eicosapentaenoic acid (EPA) and docosahexaenoic acid (DHA), to compete with arachidonic acid in the orthodox pathways of eicosanoid synthesis (Figure 1). Further support for eicosanoid-mediated n-3 PUFA effects has come from the discovery of an exciting new family of EPA/ DHA-derived eicosanoids by Serhan and colleagues [3]. 
Figure I. Pathways of enzymatic and non-enzymatic n-3 PUFA oxidation

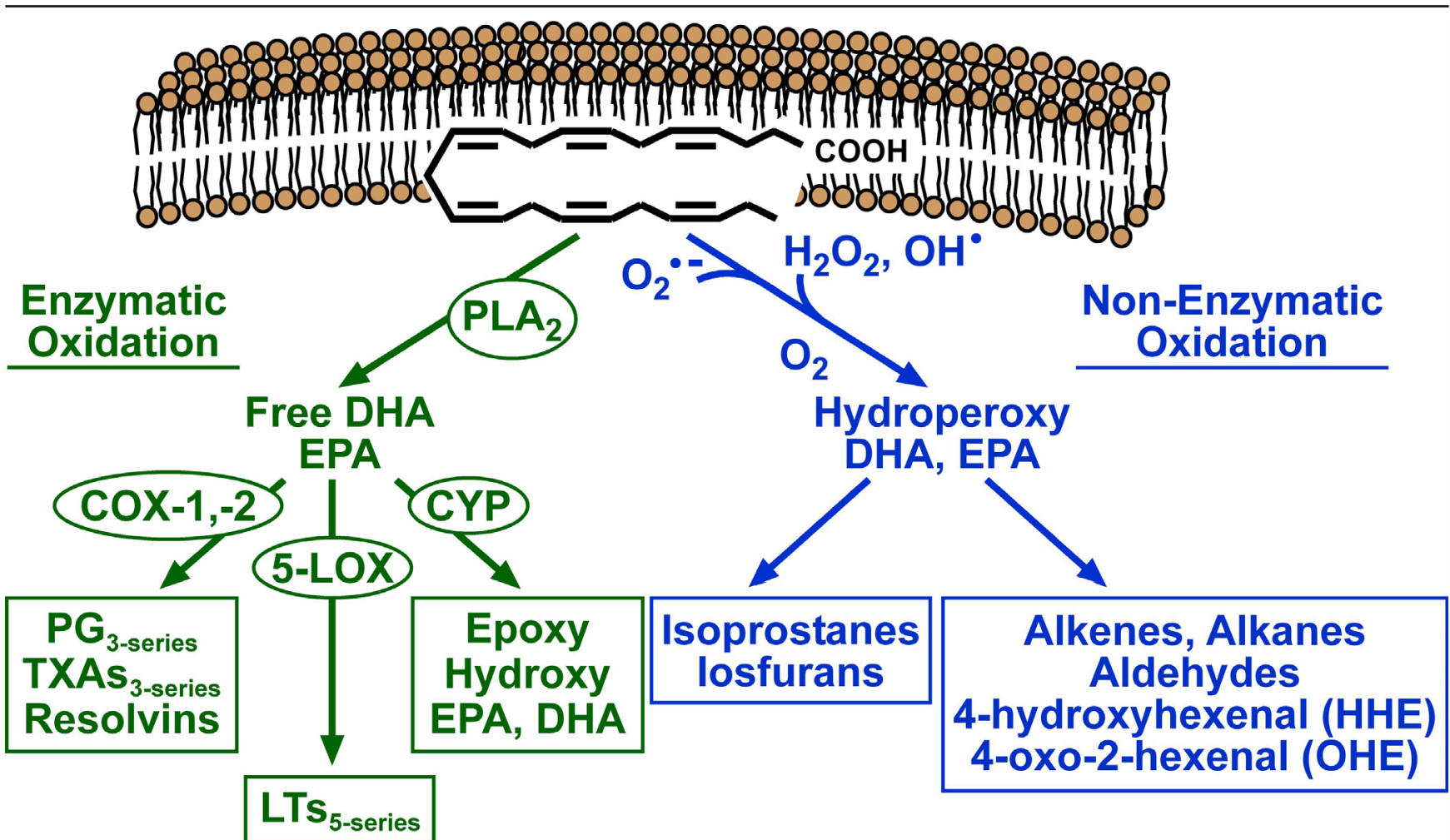

The pathway shown in green represents the orthodox enzymatic oxidation cascade that begins with the liberation of EPA or DHA from phospholipids in cellular membranes by phospholipase A2 (PLA2). The free fatty acid is then acted on by members of the cyclo-oxygenase (COX), 5-lipoxygenase (5-LOX) and cytochrome P450 monooxygenase (CYP) family of enzymes to form eicosanoids, all of which have various roles in vascular function and innate immunity. The pathway shown in blue depicts spontaneous oxidation of EPA or DHA by reactive oxygen species, such as superoxide $\left(\mathrm{O}_{2}{ }^{\circ}\right), \mathrm{H}_{2} \mathrm{O}_{2}$ and hydroxyl radical $\left(\mathrm{OH}^{\circ}\right)$. Further oxidation yields lipid peroxides of EPA or DHA which, if not neutralized by endogenous antioxidant systems, undergo destabilization and fragmentation to yield reactive lipids such as isoprostanes, isofuranes, alkenes, alkanes and aldehydes. PG, prostaglandin; TXA, thromboxane; LT, leukotriene.

This family of compounds, called the specialized proresolving mediators, exemplified by the 'resolvin' series, were found to have potent properties that enhance the resolution phase of inflammation, in addition to other potential therapeutic effects (e.g. analgesia [4]). However, while the cardiovascular benefits of EPA/DHA-derived eicosanoids must be recognized, burgeoning experimental evidence suggests that attributing the mechanism of n-3 PUFA therapy in cardiovascular disease solely to eicosanoid-mediated effects is grossly over-simplistic.

One of the more intriguing and promising therapeutic potentials for $\mathrm{n}-3$ PUFAs is in the treatment and prevention of heart failure [5]. Indeed, fish oil (DHA in particular) has been shown in several heart failure models to improve cardiac function and efficiency [6-9]. These findings have been supported recently by placebocontrolled clinical trials showing that daily intake of DHA/EPA for $\geq$ one year improved left ventricular function [10] and exercise capacity [11] in patients with established heart failure. Furthermore, clinically significant changes in left ventricular function have been reported as early as three months after initiating n-3 PUFA treatment [12]. Given that heart failure is known to result from altered cardiac energetic and structural parameters, the effects of n-3 PUFAs in enhancing these parameters has come under vigorous scrutiny by researchers.

It is clear that n-3 PUFAs are incorporated into membrane phospholipids in vivo. This is particularly important in highly oxidative, excitable tissues such as the heart, where phospholipid composition is critical for proper membrane structure, thereby ensuring that ion channel activity, charge separation and energy conservation are maintained. Several studies have proposed that EPA and DHA directly modify cardiomyocyte plasma membrane ion channel activity, which may partially explain their anti-arrhythmic properties [13-16]. Mitochondria are highly reliant on cardiolipin, a phospholipid unique to this organelle, to 
maintain membrane electrochemical gradient and capacity for oxidative phosphorylation. Recent findings in animal models of heart failure have demonstrated that altered cardiolipin structure parallels the increase in left ventricular function seen with EPA/DHA treatment [17-19], suggesting that n-3 PUFA incorporation into cardiolipin may partially explain the improvements in cardiac function. However, from a biochemical/biophysical perspective, it is not clear how altering cardiolipin structure with EPA/DHA incorporation would enhance mitochondrial function and improve cardiac energetics. Consequently, alternative metabolic pathways and roles for $n-3$ PUFAs in the heart continue to be explored. In particular, there is compelling evidence that n-3 PUFAs may play a significant role in gene regulation [20-22], which may contribute to the many beneficial effects that have been observed.

\section{Lipoxidative stress from n-3 PUFAs and adaptation in heart}

Aside from the well-characterized enzymatic pathways, PUFAs are prone to oxidation from non-enzymatic (i.e. spontaneous) reactions because of their highly unsaturated structure. These reactions, initiated by reactive oxygen and nitrogen species, ultimately form lipoxidative products such as lipid peroxides, reactive aldehydes and other electrophilic lipids (Figure 1). Of all PUFAs, DHA is the most susceptible to lipid peroxidation due to its chemical structure [23]. To date, non-enzymatic oxidation pathways and lipoxidative products of $n-3$ PUFAs have been largely ignored by investigators, with a few exceptions. The reasons for this paucity of investigation are not completely clear, but could be due to either the strongly supported belief that the rate of nonenzymatic n-3 PUFA oxidation in vivo is negligible, or that the previously held idea that any form of lipid peroxidation is undesirable as it is unconditionally toxic. The latter idea has recently been challenged on the basis of studies described below.

In fact, evidence is accumulating that suggests that PUFAderived 'lipoxidative stress' can be beneficial in many contexts, particularly in cells and tissues that are highly plastic and adaptable, such as the heart. Indeed, it has long been known that 4-hydroxynonenal, an aldehyde formed from n-6 PUFA oxidation, exerts bi-directional effects on the heart, characterized by a beneficial "hormetic" effect at sub-toxic concentrations $(\leq 10 \mu \mathrm{M})$, but toxic effects at higher concentrations [24]. The molecular mediators of these positive adaptations to "hormetic" concentrations of 4-hydroxynonenal are only beginning to be elucidated, but recent studies have pointed to the involvement of the eukaryotic translation initiating factor 2 /activating transcription factor-4 (eIF2 $\alpha /$ ATF4), NF E2-related factor-2 (Nrf2), and peroxisome proliferator-activated receptor
(PPAR) family of transcription factors in up-regulating amino acid biosynthesis [25], antioxidant/anti-inflammatory genes $[26,27]$, and mitochondrial biogenesis $[28,29]$, respectively.

Consistent with this, Gao and co-workers demonstrated that oxidized derivatives of n-3 PUFAs up-regulate Nrf2 activity in several stable cell lines, in vitro [30]. Whether similar pathways of adaptation occur in heart as a result of lipoxidative products formed from n-3 PUFAs remains to be determined (Figure 2), but a recent study in our laboratory showed that dietary intake of n-3 PUFAs resulted in a time-dependent increase in 4-hydroxyhexenal adducts in the heart and up-regulation of Nrf2-mediated enzymes in mice that were paralleled by decreased mitochondrial reactive oxygen species production and enhanced mitochondrial tolerance to insults such as $\mathrm{Ca}^{2+}$ overload [31]. Several clinical studies have reported marked increases in antioxidant enzymes such as superoxide dismutase, catalase and glutathione peroxidase in blood of patients taking n-3 PUFAs [32-37] and in some cases, lipid peroxidation levels increased in parallel with elevation in these enzymes [38,39]. Furthermore, a number of studies in animals have reported increased expression of antioxidant/anti-inflammatory enzymes in heart following n-3 PUFA diet [40-43], and these were supported by findings from a small clinical trial showing that pre-surgical intake of fish oil suppressed NFkB activity and augmented antioxidant activity in heart tissue of patients undergoing cardiac surgery [44].

\section{Treating the heart with stress: are lipoxidative products of n-3 PUFAs mediators of their therapeutic effect?}

The context and background provided by the findings outlined above allude to a provocative question: is it possible that, to some (or even a large) extent, the cardiovascular benefits derived from n-3 PUFAs result from the 'lipoxidative stress' that they cause? If so, it would follow that any physiological state resulting in sustained increases in cellular and tissue reactive oxygen species (e.g. cardiovascular and metabolic diseases) would drive increased lipoxidative product formation when presented with EPA and DHA. This is undoubtedly a controversial hypothesis because it contradicts existing paradigms regarding reactive oxygen species and disease, but we offer the following evidence in support of this.

First, recent reports demonstrated that oxidized DHA has the highest affinity and PPAR-activating effect of any PPAR ligand tested, including all members of the fibrate and glitazone drug classes $[45,46]$. This finding could have broad clinical implications because it indicates that DHA peroxidation in vivo would greatly enhance its potency as a PPAR activator. Secondly, an 
Figure 2. Proposed intracellular effects of n-3 PUFA-derived peroxides and reactive aldehydes

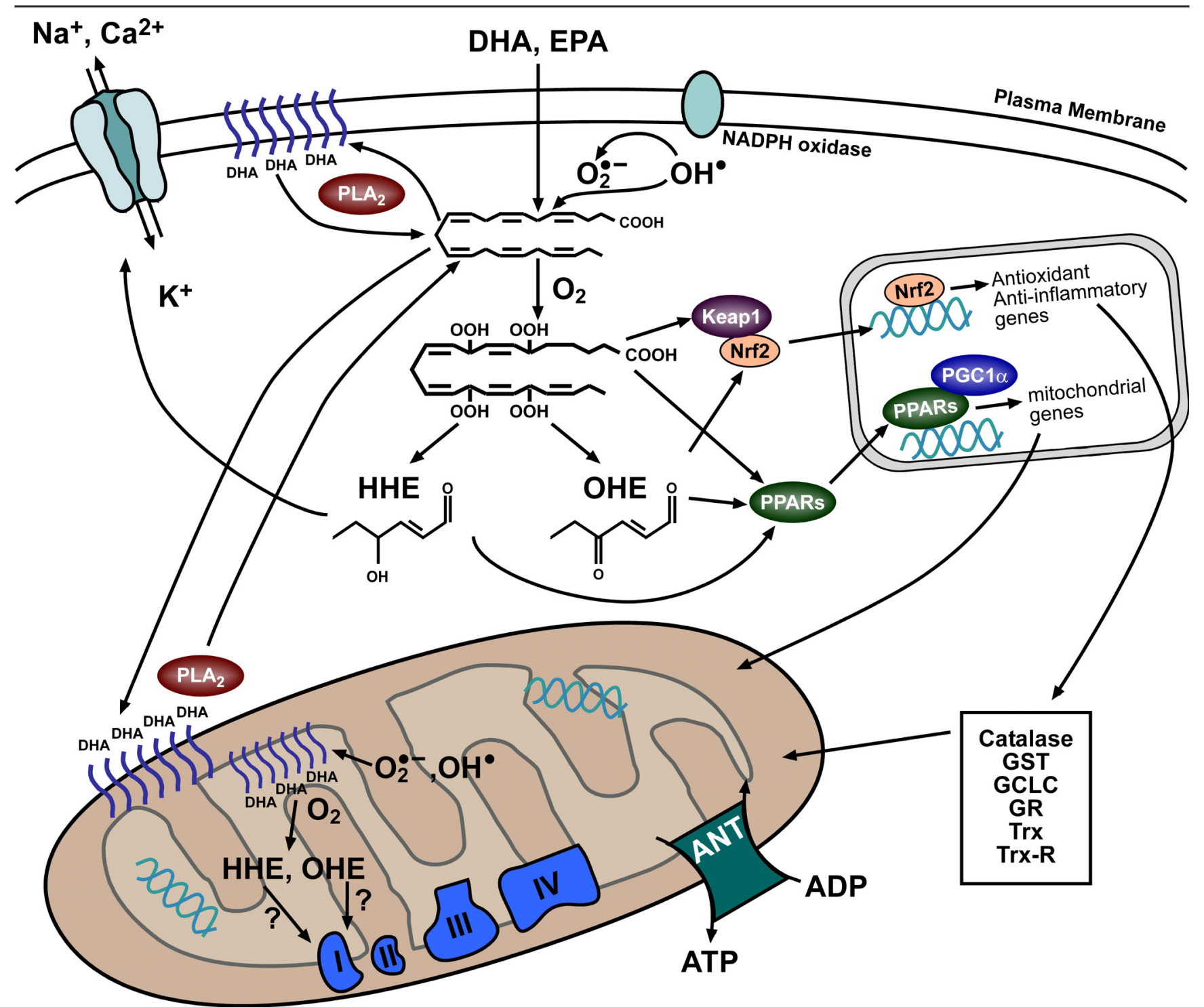

Upon entering cells, DHA and EPA are either used for oxidative metabolism (not shown) or esterified and used for phospholipids within membranes (shown here in both plasma and mitochondrial membranes). Liberation of DHA and EPA from the phospholipids is catalyzed by Phospholipase A2 (PLA2). In their free fatty acid form, the n-3 PUFAs are oxidized directly by reactive oxygen species coming from sources such as nicotinamide adenine dinucleotide phosphate (NADPH) oxidase or mitochondrial electron transport system. The lipid peroxides formed in this manner are then more reactive and their potency as transcriptional activators is increased, as depicted by the hydroperoxy-DHA reacting with PPARs and Nrf2. Sustained increases of these lipid peroxides can lead to a build-up of their reactive aldehyde derivatives such as 4-hydroxyhexenal and 4-oxo-2-hexenal (OHE), and these can also cause transcriptional activation or react with functional proteins directly. Activation of PPARs would be expected to cause increased mitochondrial gene expression and lipid oxidation capacity, particularly in the heart. Nrf2 activation would lead to increased expression of antioxidant/anti-inflammatory genes. Possible outcomes of the direct protein modifications include changes in ion channel conductance (shown in plasma membrane above) or in altered activity of mitochondrial respiratory enzymes. KeapI, Kelch-like ECH-associated protein I; PGCl $\alpha$, PPAR $\gamma$ coactivator-I $\alpha$; ANT, adenine nucleotide translocase; GST, glutathione S-transferase; GCLC, $\gamma$-glutamylcystein ligase catalytic subunit; GR, glutathione reductase; Trx, thioredoxin; Trx-R; thioredoxin reductase.

interesting study by Judé and colleagues showed that the electrophysiological effects of DHA on the transient outward current in cardiomyocytes were only present when the DHA was oxidized [47]. This finding led the authors to speculate that perhaps much of the electrophysiological effects that investigators have attributed to DHA were actually coming from lipoxidative products derived from it, since a large amount 
of DHA oxidation occurs spontaneously upon exposure to air $(\sim 30 \%)$.

As mentioned above, it is now clear that 4-hydroxynonenal can exert a beneficial effect on the heart. Zhang et al. [26] recently showed that treating cardiomyocytes with small, sub-toxic doses $(5 \mu \mathrm{M})$ of 4 -hydroxynonenal elicits protection from subsequent exposure to toxic doses $(\geq 20 \mu \mathrm{M})$. This group further showed the physiological relevance of this effect by pre-treating mice with 4-hydroxynonenal prior to ischemia/reperfusion, and showed that 4-hydroxynonenal-treated mice exhibited reduced infarct size. In addition, this 4-hydroxynonenal-induced cardioprotection was lost in Nrf2 -/- mice, suggesting a fundamental requirement for $\mathrm{Nrf} 2$ in the 4-hydroxynonenal effect [26]. The role of Nrf2 in cardioprotection is only beginning to be understood, and this was underscored by a recent study where overexpression of Nrf2 protected against

Figure 3. Diagram of proposed clinical effects of n-3 PUFA-derived peroxides and reactive aldehydes in heart

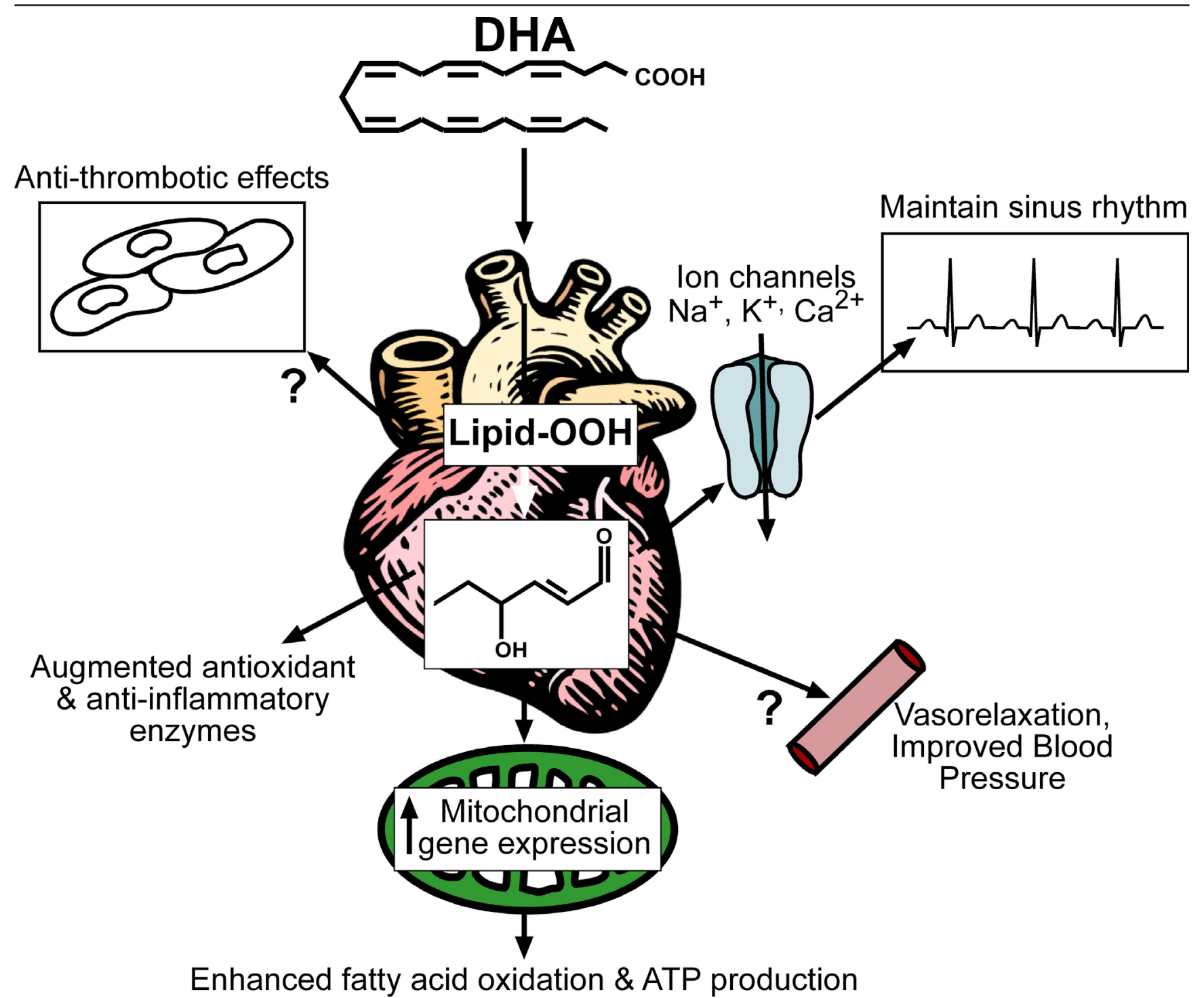

Many of the cardiovascular effects of n-3 PUFAs have been linked to their anti-thrombotic, antioxidant/anti-inflammatory, anti-arrhythmic, and vasorelaxing activities. We propose that many of these effects are mediated by n-3 PUFA-derived peroxides and reactive aldehydes. Moreover, to this list of well-known clinical effects of $n-3$ PUFAs we add enhanced mitochondrial biogenesis (i.e. gene expression) and antioxidant capacity in the myocardium, which would be expected to augment fatty acid oxidation and protect mitochondria against insults such as $\mathrm{Ca}^{2+}$ overload. The latter effects would be expected to have particular importance in clinical scenarios such as pathological cardiac hypertrophy and heart failure, conditions known to result in, or be a result of, mitochondrial dysfunction. 
pressure overload-induced cardiac hypertrophy [48]. 4-hydroxyhexenal is the n-3 PUFA alkenal equivalent to 4-hydroxynonenal, which is similar in structure but displays different reactivity. As an electrophile, it is equipotent in activating Nrf2 [27], but studies on heart mitochondria in our laboratory have shown that at physiological concentrations, 4-hydroxynonenal inhibits oxidative phosphorylation and also causes increased susceptibility to $\mathrm{Ca}^{2+}$ overload, whereas 4-hydroxyhexenal does not (unpublished data). From a translational perspective, this observation is important because it implies that n-3 PUFA-derived lipoxidative products may cause similar adaptations to those of n-6 PUFA-derived lipoxidative products, without as much of the corresponding cellular and mitochondrial toxicity.

\section{Further direction}

It must be emphasized that if indeed $n-3$ PUFA-derived lipoxidative products are partially responsible for the therapeutic effects observed, this would be manifested to the greatest extent in highly plastic and adaptable organs (i.e. organs with large antioxidant capacities, like the heart). The reason for this is because 4-hydroxyhexenal, and indeed all n-3 PUFA-derived lipoxidative products, will affect tissues and organ systems differently depending on their ability to positively adapt to mild 'lipoxidative stress.' Moreover, it is expected that this adaptation would require several days or weeks to become optimal. Despite these considerations, our contention is that when taken together, the experimental evidence outlined here and elsewhere $[49,50]$ supports the notion that many of the broad effects of n-3 PUFAs in cardiovascular disease (Figure 3) can be explained by lipoxidative products derived from them, particularly reactive aldehydes such as 4-hydroxyhexenal. If further investigation confirms this observation, it is deserving of rigorous evaluation as it may allow for future development of novel n-3 PUFA therapies in cardiovascular disease and other diseases, and assist in developing relevant and proper dosing strategies for n-3 PUFA use in clinics.

\section{Abbreviations}

DHA, docosahexaenoic acid; EPA, eicosapentaenoic acid; PPAR, proliferator-activated receptor; PUFA, polyunsaturated fatty acid.

\section{Competing interests}

The authors declare that they have no competing interests.

\section{Acknowledgements}

Supported in part by National Institutes of Health grant HL098780 to E.J.A. The authors would like to thank Kathleen Thayne for her kind assistance with preparation of Figures.

\section{References}

I. Mozaffarian D, Wu JH: Omega-3 Fatty acids and cardiovascular disease: effects on risk factors, molecular pathways, and clinical events. J Am Coll Cardiol 20I I, 58:2047-2067.

2. De Caterina R: $\mathbf{n - 3}$ fatty acids in cardiovascular disease. $N$ Engl J Med 20II, 364:2439-2450.

FI000 Factor 10

David Taylor and Ethan Anderson 20 Jun 2012

3. Serhan $\mathrm{CN}$ : Novel lipid mediators and resolution mechanisms in acute inflammation: to resolve or not? Am J Pathol 2010, I77:|576-|59|.

4. Xu ZZ, Zhang L, Liu T, Park JY, Berta T, Yang R, Serhan CN, Ji RR: Resolvins RvEl and RvDI attenuate inflammatory pain via central and peripheral actions. Nat Med 2010, 16:592-597, 59 I following 597.

\section{FI000 Factor 18}

Karsten Gronert and Elvira Liclican 09 Jun 2010, Theodore Cummins 15 Jun 2010, Mark Millan 06 Aug 2010, Bruce Levy and Mohib Uddin 16 Aug 2010, Claudia Sommer 20 Sep 2011

5. Duda MK, O'Shea KM, Stanley WC: omega-3 polyunsaturated fatty acid supplementation for the treatment of heart failure: mechanisms and clinical potential. Cardiovasc Res 2009, 84:33-4I.

6. Duda MK, O'Shea KM, Tintinu A, Xu W, Khairallah RJ, Barrows BR, Chess DJ, Azimzadeh AM, Harris WS, Sharov VG, et al.: Fish oil, but not flaxseed oil, decreases inflammation and prevents pressure overload-induced cardiac dysfunction. Cardiovasc Res 2009, 8 I:319-327.

7. McLennan PL, Barnden LR, Bridle TM, Abeywardena MY, Charnock JS: Dietary fat modulation of left ventricular ejection fraction in the marmoset due to enhanced filling. Cardiovasc Res 1992, 26:87|-877.

8. Pepe S, McLennan PL: Cardiac membrane fatty acid composition modulates myocardial oxygen consumption and postischemic recovery of contractile function. Circulation 2002, 105:2303-2308.

9. Charnock JS, McLennan PL, Abeywardena MY: Dietary modulation of lipid metabolism and mechanical performance of the heart. Mol Cell Biochem 1992, I I6:19-25.

10. Ghio S, Scelsi L, Latini R, Masson S, Eleuteri E, Palvarini M, Vriz O, Pasotti M, Gorini M, Marchioli R, et al.: Effects of $\mathbf{n}-\mathbf{3}$ polyunsaturated fatty acids and of rosuvastatin on left ventricular function in chronic heart failure: a substudy of GISSI-HF trial. Eur J Heart Fail 2010, 12:1345-1353.

FI000 Factor 8

David Taylor and Ethan Anderson 20 Jun 2012

II. Nodari S, Triggiani M, Campia U, Manerba A, Milesi G, Cesana BM, Gheorghiade M, Dei Cas L: Effects of n-3 Polyunsaturated Fatty Acids on Left Ventricular Function and Functional Capacity in Patients With Dilated Cardiomyopathy. J Am Coll Cardiol 20I I, 57:870-879.

FI000 Factor 8

David Taylor and Ethan Anderson 20 Jun 2012

12. Moertl D, Hammer A, Steiner S, Hutuleac R, Vonbank K, Berger R: Dose-dependent effects of omega-3-polyunsaturated fatty acids on systolic left ventricular function, endothelial function, and markers of inflammation in chronic heart failure of nonischemic origin: a double-blind, placebo-controlled, 3-arm study. Am Heart J 20II, I6I(9I5):e9l I-9I9.

FI000 Factor 6

David Taylor and Ethan Anderson 20 Jun 2012

13. Dart C: Lipid microdomains and the regulation of ion channel function. J Physiol 2010, 588:3169-3178. 
14. Gawrisch K, Soubias O: Structure and dynamics of polyunsaturated hydrocarbon chains in lipid bilayers-significance for GPCR function. Chem Phys Lipids 2008, 153:64-75.

15. Ferrier GR, Redondo I, Zhu J, Murphy MG: Differential effects of docosahexaenoic acid on contractions and L-type $\mathrm{Ca2}+$ current in adult cardiac myocytes. Cardiovasc Res 2002, 54:60I-6I0.

16. Li GR, Sun HY, Zhang XH, Cheng LC, Chiu SW, Tse HF, Lau CP: Omega- 3 polyunsaturated fatty acids inhibit transient outward and ultra-rapid delayed rectifier $\mathrm{K}+$ currents and $\mathrm{Na}+$ current in human atrial myocytes. Cardiovasc Res 2009, 81:286-293.

FI000 Factor 6

David Taylor and Ethan Anderson 20 Jun 2012

17. Shah KB, Duda MK, O'Shea KM, Sparagna GC, Chess DJ, Khairallah RJ, Robillard-Frayne I, Xu W, Murphy RC, Des Rosiers C, Stanley WC: The cardioprotective effects of fish oil during pressure overload are blocked by high fat intake: role of cardiac phospholipid remodeling. Hypertension 2009, 54:605-6I I.

18. O'Shea KM, Khairallah RJ, Sparagna GC, Xu W, Hecker PA, RobillardFrayne I, Des Rosiers C, Kristian T, Murphy RC, Fiskum G, Stanley WC: Dietary omega-3 fatty acids alter cardiac mitochondrial phospholipid composition and delay $\mathrm{Ca2+-induced} \mathrm{permeabil-}$ ity transition. J Mol Cell Cardiol 2009, 47:819-827.

19. Khairallah RJ, Sparagna GC, Khanna N, O'Shea KM, Hecker PA, Kristian T, Fiskum G, Des Rosiers C, Polster BM, Stanley WC: Dietary supplementation with docosahexaenoic acid, but not eicosapentaenoic acid, dramatically alters cardiac mitochondrial phospholipid fatty acid composition and prevents permeability transition. Biochim Biophys Acta 2010, I797:1555-1562.

FI000 Factor 6

David Taylor and Ethan Anderson 20 Jun 2012

20. Adkins $Y$, Kelley DS: Mechanisms underlying the cardioprotective effects of omega-3 polyunsaturated fatty acids. J Nutr Biochem 2010, 21:781-792.

FI000 Factor 6

David Taylor and Ethan Anderson 20 Jun 2012

21. Vanden Heuvel JP: Cardiovascular disease-related genes and regulation by diet. Curr Atheroscler Rep 2009, I I:448-455.

FI000 Factor 6

David Taylor and Ethan Anderson 20 Jun 2012

22. Kuang YL, Eric Paulson K, Lichtenstein AH, Lamon-Fava S: Regulation of the expression of key genes involved in HDL metabolism by unsaturated fatty acids. Br J Nutr 2012, I-9.

FI000 Factor 6

David Taylor and Ethan Anderson 20 Jun 2012

23. Siddiqui RA, Harvey K, Stillwell W: Anticancer properties of oxidation products of docosahexaenoic acid. Chem Phys Lipids 2008, 153:47-56.

FI000 Factor 6

David Taylor and Ethan Anderson 20 Jun 2012

24. Uchida K, Shiraishi M, Naito Y, Torii Y, Nakamura Y, Osawa T: Activation of stress signaling pathways by the end product of lipid peroxidation. 4-hydroxy-2-nonenal is a potential inducer of intracellular peroxide production. J Biol Chem 1999, 274:2234-2242.

FI000 Factor 6

David Taylor and Ethan Anderson 20 Jun 2012

25. Endo J, Sano M, Katayama T, Hishiki T, Shinmura K, Morizane S, Matsuhashi T, Katsumata $Y$, Zhang $Y$, Ito $H$, et al.: Metabolic remodeling induced by mitochondrial aldehyde stress stimulates tolerance to oxidative stress in the heart. Circ Res 2009, 105: I II 8-III27.

FI000 Factor 10

David Taylor and Ethan Anderson 20 Jun 2012

26. Zhang $Y$, Sano M, Shinmura K, Tamaki K, Katsumata Y, Matsuhashi T, Morizane S, Ito H, Hishiki T, Endo J, et al.: 4-hydroxy-2-nonenal protects against cardiac ischemia-reperfusion injury via the Nrf2-dependent pathway. J Mol Cell Cardiol 2010, 49:576-586.

FI000 Factor 8

David Taylor and Ethan Anderson 20 Jun 2012

27. Ishikado A, Nishio Y, Morino K, Ugi S, Kondo H, Makino T, Kashiwagi A, Maegawa $\mathrm{H}$ : Low concentration of 4-hydroxy hexenal increases heme oxygenase-I expression through activation of Nrf2 and antioxidative activity in vascular endothelial cells. Biochem Biophys Res Commun 2010, 402:99-104.

FI000 Factor 6

David Taylor and Ethan Anderson 20 Jun 2012

28. Coleman JD, Prabhu KS, Thompson JT, Reddy PS, Peters JM, Peterson BR, Reddy CC, Vanden Heuvel JP: The oxidative stress mediator 4-hydroxynonenal is an intracellular agonist of the nuclear receptor peroxisome proliferator-activated receptorbeta/delta (PPARbeta/delta). Free Radic Biol Med 2007 , 42: II55-II64.

29. Cohen G, Riahi Y, Shamni O, Guichardant M, Chatgilialoglu C, Ferreri C, Kaiser N, Sasson S: Role of lipid peroxidation and PPAR-delta in amplifying glucose-stimulated insulin secretion. Diabetes 201 I, 60:2830-2842.

30. Gao L, Wang J, Sekhar KR, Yin H, Yared NF, Schneider SN, Sasi S, Dalton TP, Anderson ME, Chan JY, et al:: Novel $\mathbf{n}-\mathbf{3}$ fatty acid oxidation products activate Nrf2 by destabilizing the association between Keap I and Cullin3. J Biol Chem 2007, 282:2529-2537.

FI000 Factor 6

David Taylor and Ethan Anderson 20 Jun 2012

31. Anderson EJ, Thayne K, Harris M, Carraway K, Shaikh SR: Aldehyde stress and up-regulation of Nrf2-mediated antioxidant systems accompany functional adaptations in cardiac mitochondria from mice fed $n-3$ polyunsaturated fatty acids. Biochem J $201 \mathrm{I}$.

32. Bouzidi N, Mekki K, Boukaddoum A, Dida N, Kaddous A, Bouchenak M: Effects of omega-3 polyunsaturated fatty-acid supplementation on redox status in chronic renal failure patients with dyslipidemia. J Ren Nutr 2010, 20:321-328.

FI000 Factor 6

David Taylor and Ethan Anderson 20 Jun 2012

33. Olivieri O, Negri M, De Gironcoli M, Bassi A, Guarini P, Stanzial AM, Grigolini L, Ferrari S, Corrocher R: Effects of dietary fish oil on malondialdehyde production and glutathione peroxidase activity in hyperlipidaemic patients. Scand J Clin Lab Invest 1988, 48:659-665.

FI000 Factor 6

David Taylor and Ethan Anderson 20 Jun 2012

34. Corrocher R, Ferrari S, de Gironcoli M, Bassi A, Olivieri O, Guarini P, Stanzial A, Barba AL, Gregolini L: Effect of fish oil supplementation on erythrocyte lipid pattern, malondialdehyde production and glutathione-peroxidase activity in psoriasis. Clin Chim Acta 1989, 179:121-131.

FI000 Factor 6

David Taylor and Ethan Anderson 20 Jun 2012

35. Bellisola G, Galassini S, Moschini G, Poli G, Perona G, Guidi G: Selenium and glutathione peroxidase variations induced by 
polyunsaturated fatty acids oral supplementation in humans. Clin Chim Acta 1992, 205:75-85.

FI000 Factor 6

David Taylor and Ethan Anderson 20 Jun 2012

36. Linseisen J, Hoffmann J, Lienhard S, Jauch KW, Wolfram G: Antioxidant status of surgical patients receiving TPN with an omega-3-fatty acid-containing lipid emulsion supplemented with alpha-tocopherol. Clin Nutr 2000, 19:177-I84.

37. Turley E, Wallace JM, Gilmore WS, Strain J]: Fish oil supplementation with and without added vitamin $E$ differentially modulates plasma antioxidant concentrations in healthy women. Lipids 1998, 33:1 I63-1 167.

FI000 Factor 6

David Taylor and Ethan Anderson 20 Jun 2012

38. Allard JP, Kurian R, Aghdassi E, Muggli R, Royall D: Lipid peroxidation during $n-3$ fatty acid and vitamin $E$ supplementation in humans. Lipids 1997, 32:535-54I.

39. Foulon T, Richard MJ, Payen N, Bourrain JL, Beani JC, Laporte F, Hadjian A: Effects of fish oil fatty acids on plasma lipids and lipoproteins and oxidant-antioxidant imbalance in healthy subjects. Scand J Clin Lab Invest 1999, 59:239-248.

FI000 Factor 6

David Taylor and Ethan Anderson 20 Jun 2012

40. Jahangiri A, Leifert WR, Kind KL, McMurchie EJ: Dietary fish oil alters cardiomyocyte $\mathbf{C a 2 +}$ dynamics and antioxidant status. Free Radic Biol Med 2006, 40:1592-1602.

FI000 Factor 6

David Taylor and Ethan Anderson 20 Jun 2012

4I. Anandan R, Mathew S, Sankar TV, Viswanathan Nair PG: Protective effect of $n-3$ polyunsaturated fatty acids concentrate on isoproterenol-induced myocardial infarction in rats. Prostaglandins Leukot Essent Fatty Acids 2007, 76:153-I58.

FI000 Factor 6

David Taylor and Ethan Anderson 20 Jun 2012

42. Padma VV, Devi CS, Ramkumar KM: Modulatory effect of fish oil on the myocardial antioxidant defense system in isoproterenol-induced myocardial infarction. I Basic Clin Physiol Pharmacol 2006, I7:1-15

FI000 Factor 6

David Taylor and Ethan Anderson 20 Jun 2012

43. Nalbone G, Leonardi J, Termine E, Portugal H, Lechene P, Pauli AM, Lafont $\mathrm{H}$ : Effects of fish oil, corn oil and lard diets on lipid peroxidation status and glutathione peroxidase activities in rat heart. Lipids 1989, 24:179-186.

FI000 Factor 6

David Taylor and Ethan Anderson 20 Jun 2012

44. Castillo R, Rodrigo R, Perez F, Cereceda M, Asenjo R, Zamorano J, Navarrete R, Villalabeitia E, Sanz J, Baeza C, Aguayo R: Antioxidant therapy reduces oxidative and inflammatory tissue damage in patients subjected to cardiac surgery with extracorporeal circulation. Basic Clin Pharmacol Toxicol 201 I, I 08:256-262.

FI000 Factor 8

David Taylor and Ethan Anderson 20 Jun 2012

45. Itoh T, Yamamoto K: Peroxisome proliferator activated receptor gamma and oxidized docosahexaenoic acids as new class of ligand. Naunyn Schmiedebergs Arch Pharmacol 2008, 377:54 I-547.

46. Itoh T, Fairall L, Amin K, Inaba Y, Szanto A, Balint BL, Nagy L, Yamamoto K, Schwabe JW: Structural basis for the activation of PPARgamma by oxidized fatty acids. Nat Struct Mol Biol 2008, I5:924-93|.

FI000 Factor 6

David Taylor and Ethan Anderson 20 Jun 2012

47. Jude $S$, Bedut $S$, Roger S, Pinault $M$, Champeroux $P$, White $E$, Le Guennec JY: Peroxidation of docosahexaenoic acid is responsible for its effects on I TO and I SS in rat ventricular myocytes. Br J Pharmacol 2003, 139:816-822.

48. Li J, Ichikawa T, Villacorta L, Janicki JS, Brower GL, Yamamoto M, Cui T: Nrf2 protects against maladaptive cardiac responses to hemodynamic stress. Arterioscler Thromb Vasc Biol 2009, 29: I843-I850.

FI000 Factor 6

David Taylor and Ethan Anderson 20 Jun 2012

49. Sano M: Cardioprotection by hormetic responses to aldehyde. Circ J 2010, 74: 1787-1793.

50. Anderson EJ, Katunga LA, Willis MS: Mitochondria as a Source and Target of Lipid Peroxidation Products in Healthy and Diseased Heart. Clin Exp Pharmacol Physiol 201 I. 